\title{
Astrometry of Galactic Star-Forming Regions ON1 and ON2N with VERA
}

\author{
Takumi Nagayama ${ }^{1}$ and VERA project members \\ ${ }^{1}$ Mizusawa VLBI Observatory, National Astronomical Observatory of Japan, 2-21-1 Osawa, \\ Mitaka, Tokyo, 181-8588, Japan \\ email: takumi.nagayama@nao.ac.jp
}

\begin{abstract}
We conducted the astrometry of $\mathrm{H}_{2} \mathrm{O}$ masers in the Galactic star-forming regions ON1 and ON2N with the VLBI Exploration of Radio Astrometry (VERA). The measured distances to $\mathrm{ON} 1$ and $\mathrm{ON} 2 \mathrm{~N}$ are $2.47 \pm 0.11 \mathrm{kpc}$ and $3.83 \pm 0.13 \mathrm{kpc}$, respectively. In the case that $\mathrm{ON} 1$ and $\mathrm{ON} 2 \mathrm{~N}$ are on a perfect circular rotation, we estimate the angular rotation velocity of the Galactic rotation at the Sun (the ratio of the Galactic constants) to be $28 \pm$ $2 \mathrm{~km} \mathrm{~s}^{-1} \mathrm{kpc}^{-1}$ using the measured distances and three-dimensional velocity components of ON1 and ON2N. This value is larger than the IAU recommended value of $25.9 \mathrm{~km} \mathrm{~s}^{-1} \mathrm{kpc}^{-1}$, but consistent with other results recently obtained with the VLBI technique.
\end{abstract}

Keywords. astrometry, Galaxy: fundamental parameters, Galaxy: kinematics and dynamics

\section{Introduction}

The Galactic constants, the distance from Sun to the Galactic center $\left(R_{0}\right)$ and the Galactic rotation velocity at Sun $\left(\Theta_{0}\right)$ are major parameters to study the structure of the Milky Way Galaxy (MWG). Although IAU has recommended the values of $R_{0}=8.5 \mathrm{kpc}$ and $\Theta_{0}=220 \mathrm{~km} \mathrm{~s}^{-1}$ since 1985, Recent studies report the values different from them (e.g. Reid et al. 2009). However, observational estimation of the Galactic constants is difficult. This is because the observational estimation of Galactic constants is affected by several independent assumptions; the peculiar motion of the source, systemic non-circular motions of both the source and the LSR due to the spiral arm and the non-axisymmetric potential of the MWG, and relative motion of Sun to the LSR (Reid et al. 2009; McMillan \& Binney 2010). To minimize these effects, we should observe many sources located at various positions in the MWG.

The tangent point and the Solar circle are kinematically unique positions in the MWG. We can estimate $R_{0}$ and $\Theta_{0}$ from the source proper motion and distance. This is because that in the case of the source located at tangent point, the proper motion depends only on the Galactic rotation of Sun, $\Theta_{0}$. The tangent point, Sun, and the Galactic center make the right triangle. In the case of the source on the solar circle, the proper motion depends only on $\Theta_{0}$. The source, Sun, and the Galactic center make an isosceles triangle. Even if the source is not exactly located at tangent point or on the Solar circle, but near there, we can estimate the ratio of the Galactic constants, $\Omega_{0}=\Theta_{0} / R_{0}$, as described in section 3. The radial velocity of ON1 is $12 \pm 1 \mathrm{~km} \mathrm{~s}^{-1}$ (e.g. Bronfman et al. 1996). It is close to the terminal velocity at $l=69.54^{\circ}$ of $15 \pm 5 \mathrm{~km} \mathrm{~s}^{-1}$ Dame et al. 2001 . The radial velocity of $\mathrm{ON} 2 \mathrm{~N}$ is $0 \pm 1 \mathrm{~km} \mathrm{~s}^{-1}$ (e.g. Olmi \& Cesaroni 1999). These suggest that ON1 is located near the tangent point, and ON2N is located near the Solar circle. In the present study, we report about the parallax measurements of ON1 and ON2N with VERA. This is a first step to estimate the Galactic constants using VERA. This study is published in Nagayama et al. 2011 and Ando et al. 2011. 


\section{Observations}

We observed $\mathrm{H}_{2} \mathrm{O}$ masers in the star-forming regions ON1 and ON2N with VERA at 11 epochs in 2006-2008. The position reference sources of ON1 and ON2N are J2010+3322 and $\mathrm{J} 2015+3710$, respectively. Their separation angles are $1.85^{\circ}$ and $1.27^{\circ}$, respectively. The maser sources and the position reference sources were simultaneously observed in a VERA dual-beam mode for about 10 hours. The data were recorded onto magnetic tapes at a rate of $1024 \mathrm{Mbps}$, providing a total bandwidth of $256 \mathrm{MHz}$, which consists of $16 \times 16 \mathrm{MHz}$ IF channels. One IF channel was assigned to the maser source, and the other 15 IF channels were assigned to the position reference source, respectively. Correlation processing was carried out on the Mitaka FX correlator. Data reduction was conducted using the NRAO Astronomical Image Processing System (AIPS).

\section{Results and Discussion}

Figure 1 and 2 show Parallaxes the $\mathrm{H}_{2} \mathrm{O}$ masers in $\mathrm{ON} 1$ and $\mathrm{ON} 2 \mathrm{~N}$, respectively. The positional variations show systematic sinusoidal modulation with a period of one year caused by the parallax. We conducted a combined parallax fit, in which the positions of ten features are fitted simultaneously with one common parallax but different proper motions and position offsets for each spot. The resulting parallaxes of ON1 and ON2N are $0.404 \pm 0.019$ mas $(2.47 \pm 0.11 \mathrm{kpc})$ and $0.261 \pm 0.009$ mas $(3.83 \pm 0.13 \mathrm{kpc})$, respectively. The obtained distance of $\mathrm{ON} 1$ is consistent with the $6.7 \mathrm{GHz} \mathrm{CH}_{3} \mathrm{OH}$ maser parallax corresponding to $2.57_{-0.27}^{+0.34} \mathrm{kpc}$ measured by Rygl et al. 2010. Figure 3 shows the position of $\mathrm{ON} 1$ and $\mathrm{ON} 2 \mathrm{~N}$ in the MWG. ON1 and ON2N are appeared to be located near the tangent point at $l=69.54^{\circ}$ and the Solar cirlce, respectively.

The systemic proper motions of ON1 and ON2N are derived to be $\left(\mu_{\alpha} \cos \delta, \mu_{\delta}\right)=$ $(-3.10 \pm 0.18,-4.70 \pm 0.24)$ and $(-2.79 \pm 0.13,-4.66 \pm 0.17)$ mas $\mathrm{yr}^{-1}$ from the averages of the proper motions of maser features. We convert to the proper motions with respect to LSR using the Solar motion in the traditional definition of $\left(U_{\odot}, V_{\odot}, W_{\odot}\right)=$ $(10.3,15.3,7.7) \mathrm{km} \mathrm{s}^{-1}$. The proper motion of ON1 and ON2N with respect to LSR in the direction of $l$ and $b$ are calculated to be $\left(\mu_{l}, \mu_{b}\right)=(-6.00 \pm 0.22,0.69 \pm 0.20)$ and $(-5.42 \pm 0.16,-0.36 \pm 0.14)$ mas $_{\mathrm{yr}^{-1}}$, respectively. This proper motions correspond to a velocity of $\left(v_{l}, v_{b}\right)=(-70.2 \pm 2.6,8.1 \pm 2.3)$ and $(-98.4 \pm 2.9,-6.6 \pm 2.6) \mathrm{km} \mathrm{s}^{-1}$.
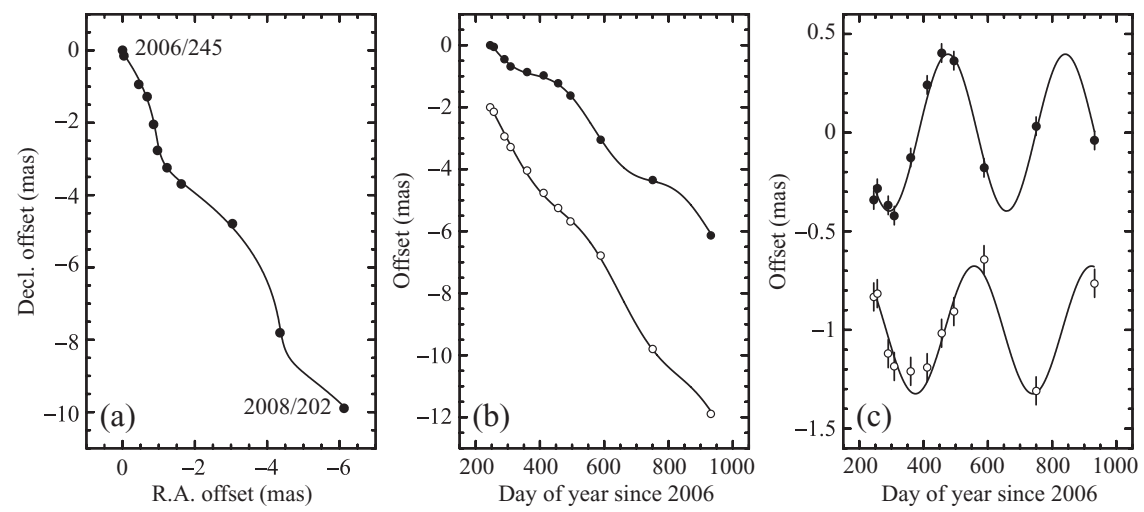

Figure 1. Parallax and proper motion data and fits of the $\mathrm{H}_{2} \mathrm{O}$ maser in ON1. (a): Positions on the sky with first and last epochs labeled. (b): $x$ (filled circles) and $y$ (open circles) position offsets versus time. (c): same as (b) panel, expect the proper motion fit has been removed, allowing the effects of only the parallax to be seen. 


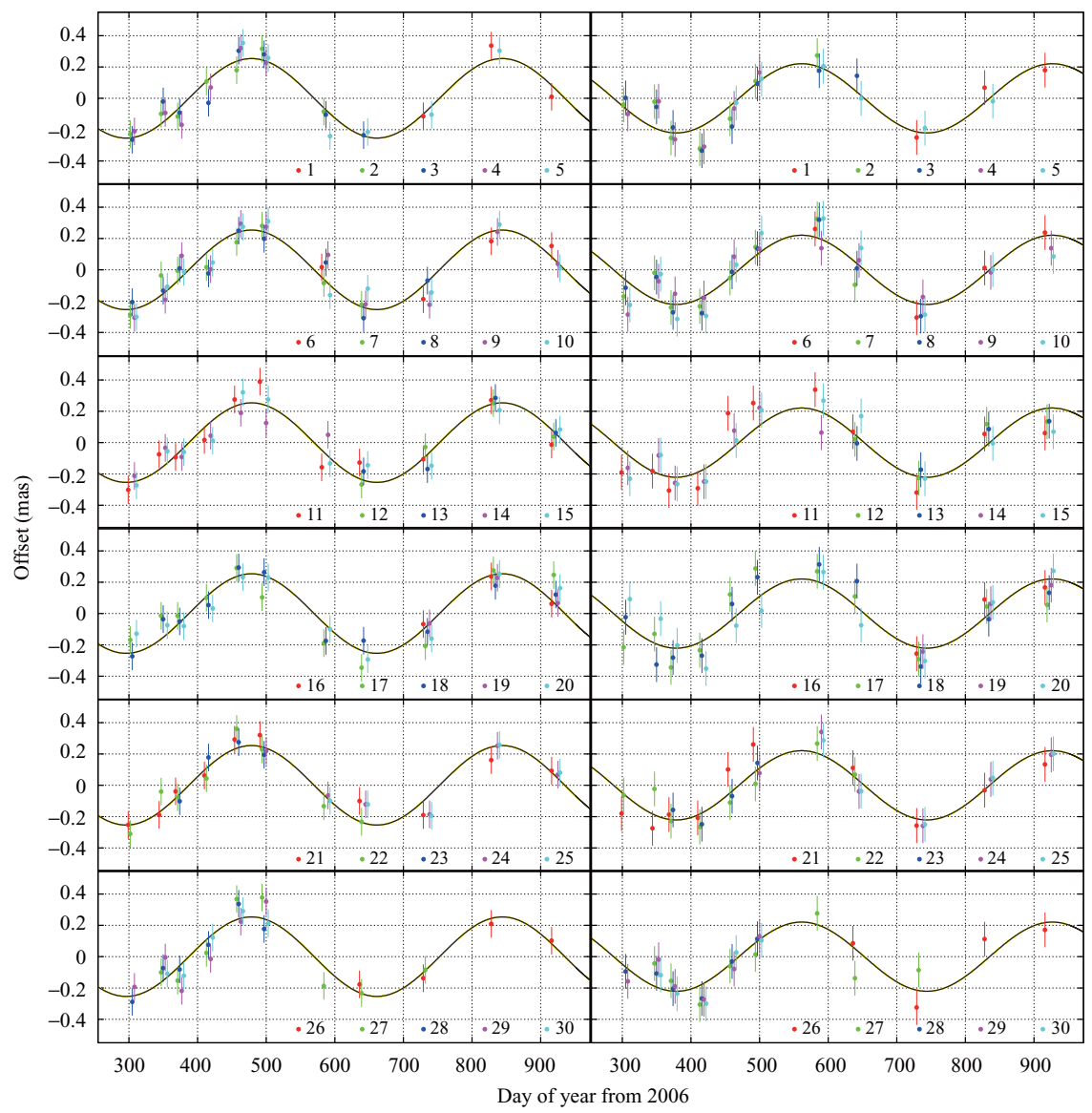

Figure 2. Parallax of the $\mathrm{H}_{2} \mathrm{O}$ masers in ON2N. Individual proper motions and position offsets are removed. The left panel shows the parallax in right ascension. The right panel shows the parallax in declination.

In the case that the source is located exactly at the tangent point, and it is on pure circular rotation, the source, Sun, and the Galactic center make the right triangle, and the source proper motion on the sky depends only on $\Theta_{0}$ This geometry is shown in Figure 4(a). Therefore, $R_{0}$ and $\Theta_{0}$ are determined from the observed distance $D$ and the proper motion along the Galactic plane, $v_{l}$, as

$$
\begin{aligned}
& R_{0}=D / \cos l \\
& \Theta_{0}=-v_{l} / \cos l .
\end{aligned}
$$

The Galactic constants are estimated to be $R_{0}=7.1 \pm 0.3 \mathrm{kpc}$ and $\Theta_{0}=201 \pm 7 \mathrm{~km} \mathrm{~s}^{-1}$, respectively, from $D=2.47 \pm 0.11 \mathrm{kpc}$ and $v_{l}=-70.2 \pm 2.6 \mathrm{~km} \mathrm{~s}^{-1}$. These values are approximately 10-20\% smaller than the IAU recommended values of $R_{0}=8.5 \mathrm{kpc}$ and $\Theta_{0}=220 \mathrm{~km} \mathrm{~s}^{-1}$, the recently estimated value of $R_{0}=8.4 \pm 0.6 \mathrm{kpc}$ and $\Theta_{0}=254 \pm 16$ $\mathrm{km} \mathrm{s}^{-1}$ (Reid et al. 2009). However, our estimated values would not be inconsistent with the previous estimates. This is because our estimation is affected by the ambiguities of the two assumptions that $\mathrm{ON} 1$ is on the pure circular rotation, and located exactly at the tangent point. If we consider the effects of this amibiguities, the errors of our estimated values increase to approximately $70 \%$ (Nagayama et al. 2011). 


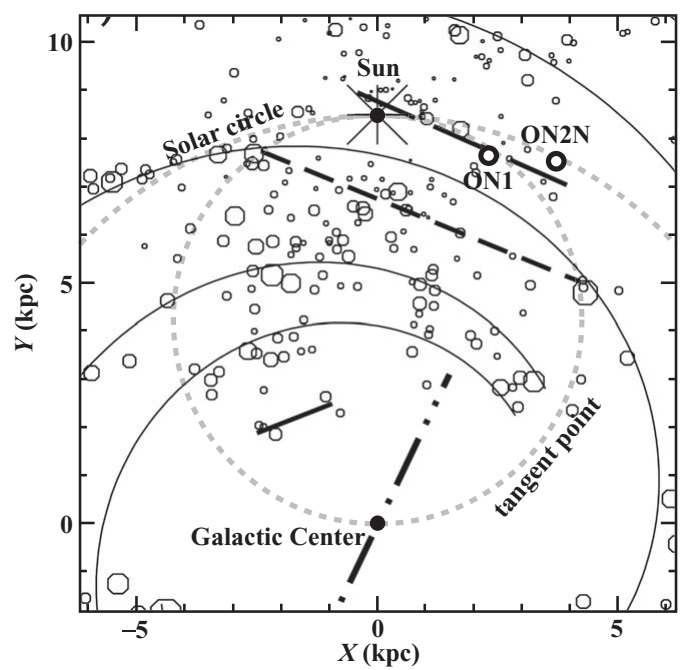

Figure 3. Positions of $\mathrm{ON} 1$ and $\mathrm{ON} 2 \mathrm{~N}$ in the MWG. The background is the four spiral arm structure of the MWG (Russeil 2003).

Although the estimated values of the Galactic constants are strongly affected by the assumption of the source location in the MWG, we found that the ratio of Galactic constants, $\Theta_{0} / R_{0}$, can be estimated with small ambiguity. In the case that the source is on pure circular rotation at any position in the Galactic disk, the radial and tangential velocities of the source can be written as

$$
\begin{aligned}
& v_{r}=\left(\frac{\Theta}{R}-\frac{\Theta_{0}}{R_{0}}\right) R_{0} \sin l, \\
& v_{l}=\left(\frac{\Theta}{R}-\frac{\Theta_{0}}{R_{0}}\right) R_{0} \cos l-\frac{\Theta}{R} D .
\end{aligned}
$$

From these equations, the relation between the $\Theta_{0}$ and $R_{0}$ is obtained to be

$$
\Theta_{0}=\left[-\frac{v_{l}}{D}+v_{r}\left(\frac{1}{D \tan l}-\frac{1}{R_{0} \sin l}\right)\right] R_{0}=\left[-a_{0} \mu_{l}+v_{r}\left(\frac{1}{D \tan l}-\frac{1}{R_{0} \sin l}\right)\right] R_{0},
$$

where $a_{0}$ is a conversion constant from a proper motion to a linear velocity $\left(4.74 \mathrm{~km} \mathrm{~s}^{-1}\right.$ $\mathrm{mas}^{-1} \mathrm{yr} \mathrm{kpc}^{-1}$ ). The equation (3.5) is graphed in Figure 5(a) using the observed values of $D=2.47 \pm 0.11 \mathrm{kpc}, \mu_{l}=-6.00 \pm 0.22 \mathrm{mas} \mathrm{yr}^{-1}$, and $v_{r}=12 \pm 1 \mathrm{~km} \mathrm{~s}^{-1}$. We found that the slope in Figure $5(\mathrm{a})$ is a nearby constant at $7 \leqslant R_{0} \leqslant 9 \mathrm{kpc}$. The slope yields the ratio $\Theta_{0} / R_{0}$, which is described as

$$
\frac{\Theta_{0}}{R_{0}}=-\frac{v_{l}}{D}+v_{r}\left(\frac{1}{D \tan l}-\frac{1}{R_{0} \sin l}\right)=-a_{0} \mu_{l}+v_{r}\left(\frac{1}{D \tan l}-\frac{1}{R_{0} \sin l}\right),
$$

The equation (3.6) is graphed in Figure 5(b). The ratio is estimated to be $\Theta_{0} / R_{0}=$ $28.7 \pm 1.3 \mathrm{~km} \mathrm{~s}^{-1} \mathrm{kpc}^{-1}$ using the above observed values, and $7 \leqslant R_{0} \leqslant 9 \mathrm{kpc}$. The error of $\Theta_{0} / R_{0}$ mainly depends on that of $\mu_{l}$. The errors of $\Theta_{0} / R_{0}$ depend on those of $v_{r}$ and $D$ are \pm 0.02 and $\pm 0.05 \mathrm{~km} \mathrm{~s}^{-1} \mathrm{kpc}^{-1}$, respectively, and they can be neglected in this estimation. This is because that $D \tan l \simeq R_{0} \sin l$ in the case that the source is located near the tangent point (see Figure 4(b)).

The ratio can be also estimated from the observed distance, proper motion, radial velocity of the source near the Solar circle. The radial velocity of the source near the solar 
circle is close to zero. Therefore, the ratio is also free from $R_{0}$. The ratio is obtained to be $\Theta_{0} / R_{0}=27.3 \pm 0.8 \mathrm{~km} \mathrm{~s}^{-1} \mathrm{kpc}^{-1}$ using the observed ON2N parameters of $D=3.83 \pm 0.13$

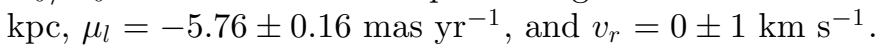

We estimated the ratio $\Theta_{0} / R_{0}=28 \pm 2 \mathrm{~km} \mathrm{~s}^{-1} \mathrm{kpc}^{-1}$ from the average of obtained values of $\mathrm{ON} 1$ and $\mathrm{ON} 2 \mathrm{~N}$. This value is consistent with the value of $\Theta_{0} / R_{0}=28.6 \pm 0.2 \mathrm{~km}$ $\mathrm{s}^{-1} \mathrm{kpc}^{-1}$ obtained from the proper motion measurement of Sgr A* (Reid \& Brunthaler 2004), which is revised using the traditional definition of the solar motion by us. However, this value is inconsistent to that derived from the IAU recommended values $220 \mathrm{~km}$ $\mathrm{s}^{-1} / 8.5 \mathrm{kpc}=25.9 \mathrm{~km} \mathrm{~s}^{-1} \mathrm{kpc}^{-1}$.

(a)

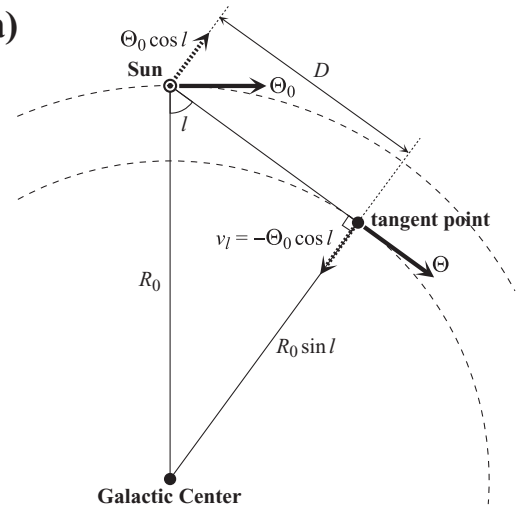

(b)

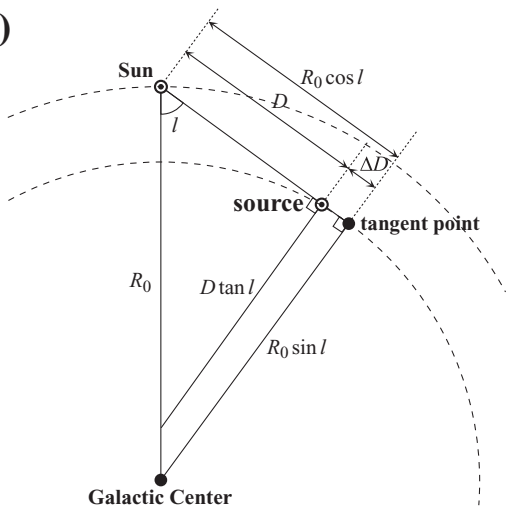

Figure 4. The geometry of the Galactic center, Sun, the tangent point, and the source. (a): The geometry in the case that the source is located at the tangent point. (b): The geometry in the case that there is a offset between the source and the tangent point.
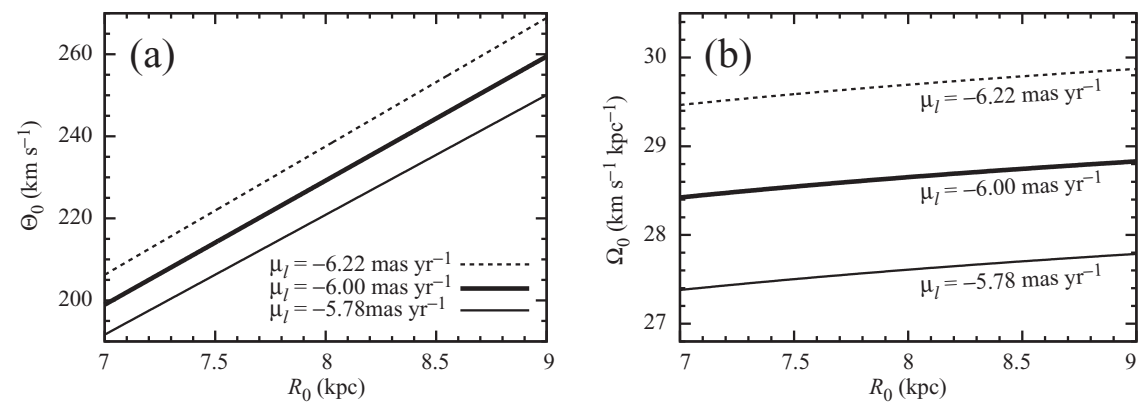

Figure 5. (a): The relation of $R_{0}$ and $\Theta_{0}$ shown in the equation (3.5). (b): The relation of $R_{0}$ and $\Theta_{0} / R_{0}$ shown in the equation (3.6).

\section{References}

Ando, K., Nagayama, T., \& Omodaka, T., et al. 2011, PASJ, 63, 45

Bronfman, L., Nyman, L.-A., \& May, J. 1996, A\&SAS, 115, 81

Dame, T. M., Hartmann, D., \& Thaddeus, P. 2001, ApJ, 547, 792

McMillan, P. J. \& Binney, J. J. 2010, MNRAS, 402, 934

Nagayama, T., Omodaka, T., Nakagawa, A., et al. 2011, PASJ, 63, 23

Olmi, L. \& Cesaroni, R. 1999, A\& A, 352, 266

Reid, M. J. \& Brunthaler, A. 2004, ApJ, 616, 872

Reid, M. J., et al. 2009, ApJ, 700, 137

Russeil, D. 2003, AESA, 397, 133

Rygl, K. L. J., Brunthaler, A., Reid, M. J., Menten, K. M., van Langevelde, H. J., \& Xu, Y. 2010, $A \mathscr{E} A, 511, \mathrm{~A} 2$ 